\title{
Cuando se hace mal: Contribuciones de los profesionales de las tecnologías museísticas en tiempos de crisis
}

\author{
Paul F. Marty ${ }^{\mathrm{a}}$, Vivian Buchanan ${ }^{\mathrm{b}}$ \\ ${ }^{\text {ab }}$ School of Information, Florida State University, USA.
}

\begin{abstract}
Resumen
Este artículo explora las contribuciones de los profesionales de las tecnologías de los museos en tiempos de crisis y analiza cómo los líderes de los museos pueden aprovechar mejor esas contribuciones. Abordando las siguientes preguntas clave: ¿Cómo pueden los profesionales de la tecnología de los museos contribuir a sus museos en tiempos de crisis? ¿Cómo ven estas contribuciones los directores/as de los museos? ¿Cómo pueden los profesionales de la tecnología de los museos defender el valor de sus contribuciones durante situaciones de crisis para mejorar el liderazgo de los museos? ¿Cómo pueden los dirigentes de los museos, y los profesionales de la tecnología, trabajar juntos para desarrollar las habilidades digitales, la mentalidad y las estrategias que todos los museos necesitarán para enfrentar nuevos desafios y sobrevivir a crisis futuras?
\end{abstract}

Palabras clave: profesionales de las tecnologías museísticas; informática de museo; museos en crisis; alfabetización digital; estrategias digitales.

\footnotetext{
Abstract

This paper explores the contributions of museum technology professionals during times of crisis and discusses how museum leadership can best take advantage of those contributions. It addresses the following key questions: How can museum technology professionals contribute to their museums during times of crisis? How are these contributions viewed by museum leadership? How can museum technology professionals advocate the value of their contributions during crisis situations to museum leadership? How can museum leadership and museum technology professionals work together to develop the digital skills, mindset, and strategies that all museums will need to meet new challenges and survive future crises?
}

Keywords: museum technology professionals; museum computing; museums in crisis; digital literacies; digital strategies 


\section{Introducción}

En marzo de 2020, cuando la pandemia de COVID-19 se extendió por todo el mundo y los países cerraron sus fronteras, los tecnólogos de los museos entraron en acción, aprovechando el poder de la informática para llegar a audiencias y comunidades que repentinamente dejaron de visitar estas instituciones en persona. El Getty Art Challenge, por ejemplo, alentó a sus audiencias y seguidores, que permanecían confinados en sus casas, a recrear sus pinturas favoritas y compartirlas en las redes sociales (McGrath, 2020). Cientos de miles de personas en todo el mundo participaron en este desafío y, según Hart et al. (2020), el tráfico al sitio web del museo Getty aumentó más del $200 \%$ en abril de 2020, y la participación de las redes sociales del museo aumentó en más del 1,500\% como resultado directo. Con el evidente éxito de proyectos como el Getty Art Challenge, parece obvio que el potencial de los profesionales de la tecnologías de los museos resulta clave para conectar con los visitantes, sin importar de qué parte del mundo procedan.

Sin embargo, hemos visto como a medida que la pandemia se prolongaba y los presupuestos de los museos iban empeorando, muchos profesionales tecnólogos han sido los primeros en ser despedidos. Como escribió Small (2020), "los déficits presupuestarios han caído en una regresión de las prioridades en muchos museos, donde campos que alguna vez crecieron, como los medios digitales y la educación, están siendo objeto de recortes". De manera similar, la Alianza Americana de Museos (2020) halló que, a pesar del valor obvio de las experiencias en línea dirigidas hacia los visitantes de los museos, dos tercios de los museos en los Estados Unidos estaban recortando en "educación, programación u otros servicios públicos". Estas decisiones frustraron naturalmente a muchos profesionales relacionados con la tecnologías museísticas. Como tuiteó Evjen (2020), "Esos son los profesionales que los museos necesitan justo ahora. Si se les está despidiendo, se está haciendo mal ".

Dada la comoplicada situación financiera que trajo la pandemia de COVID-19 (AAM, 2020; ICOM, 2020; UNESCO, 2020), algunos recortes han sido, por supuesto, inevitables. Pero los museos han resistido otras crisis anteriores y han sobrevivido. La crisis económica de 2008, por ejemplo, devastó a los museos de todo el mundo, pero finalmente se recuperaron, y algunos incluso volvieron más fuertes que nunca (Rizzo, 2010). Como observó el Consejo Internacional de Museos en 2013, los museos "fortalecen las identidades culturales, apoyan la cohesión social y desarrollan la mediación intercultural, actividades que son fundamentales en tiempos de crisis" (ICOM, 2013). ¿Es que no podemos aprender de nuestro pasado para planificar nuestro futuro?. En lugar de despedir a las personas que están en mejores condiciones para ayudarnos a superar las crisis, ¿no podemos reconocer el papel que desempeñan los profesionales de las tecnologías de los museos en las crisis? En resumen, ¿podemos dejar de "hacerlo mal"?

Para explorar las contribuciones de los profesionales relacionados con las tecnologías de los museos en tiempos de crisis y discutir cómo los líderes y dirigentes de museos pueden aprovechar mejor esas contribuciones, este artículo aborda las siguientes cuatro cuestiones: ¿Cómo pueden los profesionales de las tecnologías contribuir a sus museos en tiempos de 
crisis? ¿Cómo ven estas contribuciones los dirigentes de los museos? ¿Cómo pueden estos profesionales defender el valor de sus contribuciones durante situaciones de crisis para lograr un mayor liderazgo de sus museos? ¿Cómo pueden los dirigentes y los tecnólogos trabajar juntos para desarrollar las habilidades digitales, mentalidad y estrategias que todos los museos necesitarán con el fín de enfrentar nuevos desafíos y sobrevivir a crisis futuras?.

\section{Identificación de las contribuciones de los profesionales de la tecnologías de los museos}

En los tiempos de crisis, los profesionales relacionados con la tecnología pueden contribuir de muchas maneras diferentes. Al centrarse en la participación de la audiencia, los medios digitales y la divulgación en línea, tienen la oportunidad de ayudar a sus instituciones a encontrar nuevas formas para continuar siendo relevantes y estar conectados con sus comunidades, particularmente en situaciones de crisis en las que las audiencias no pueden visitar los museos físicamente. En particular, cuando las crisis obligan a los museos a cerrar sus puertas al público, brindar acceso a la información, llegar a nuevas audiencias a través de nuevos medios e involucrar a esas audiencias con actividades en línea se convierten en acciones de vital importancia para los profesionales de los museos, especialmente porque cada vez más visitantes esperan que precisamente los museos ofrezcan este tipo de interacciones como parte de la experiencia de la visita.

Como argumenta Anderson (2018), “el uso de nuevas tecnologías dentro de estos procesos es cada vez más importante para reconceptualizar al público del museo y a los visitantes, particularmente a medida que los medios se entrelazan firmemente en la vida de las personas y las experiencias con el museo" (p.81). Por ejemplo, al proporcionar acceso remoto a la información relacionada con las colecciones, el museo puede ofrecer nuevas oportunidades para que sus profesionales interactúen con los visitantes fuera del museo, convirtiendo las interacciones unidireccionales sincrónicas ubicadas en los museos en interacciones asincrónicas bidireccionales que pueden tener lugar en cualquier parte del mundo. De manera similar, existe la posibilidad de involucrar al público a través de actividades en línea que sean relevantes para su vida cotidiana, ofreciendo una nueva oportunidad de expandir el alcance del museo hacia diferentes comunidades, incluyendo a las personas que probablemente nunca antes hubieran interactuado en un museo, con o sin crisis.

Los profesionales de las tecnologías también pueden contribuir internamente en tiempos de crisis, es precisamente entonces cuando su experiencia con las tecnologías de la información y la comunicación puede resultar más útil. Por ejemplo, al respaldar la infraestructura técnica que subyace en las operaciones museísticas (la misma tecnología que, entre otras cosas, permite a los empleados trabajar desde casa) y salvaguardar la integridad de los recursos, sistemas y tecnologías de información. Se trata de roles con gran importancia. Del mismo modo, estos profesionales probablemente participarán directamente en el desarrollo y mantenimiento del plan estratégico del museo para definir operaciones digitales durante situaciones de crisis (es muy probable que incluso adapten el plan estratégico del museo sobre 
la marcha, a medida que evoluciona la crisis), y también pueden responsabilizarse de documentar la situación, para dar respuesta desde el museo a la crisis a largo plazo.

Estos profesionales pueden ofrecer acceso a la información de las colecciones, involucrar al público a través de actividades en línea y satisfacer las necesidades de información dentro y fuera del museo, demostrando ser vitales incluso en las situaciones de crisis, especialmente en la era digital. A medida que se desarrollaba la pandemia de COVID-19, museo tras museo buscaban empleados que pudieran ayudarles a implementar estrategias digitales necesarias para sobrevivir a la crisis, siendo los tecnológos de los museos quienes desarrollaron estas estrategias, construyendo la infraestructura y promoviendo las tecnologías digitales necesarias. Si las contribuciones de los profesionales de las tecnologías pudieran ser reconocidas, ayudarían a los museos a sobrellevar nuevas crisis. Además, éstas deberían estar claramente documentadas y ser aclaradas para todas las partes interesadas.

\section{Reconocimiento de las contribuciones de los profesionales de las tecnologías de los museos}

A pesar de los beneficios que ofrencen las contribuciones de los profesionales relacionados con la tecnología de los museos en tiempos de crisis, estas contribuciones pueden pasar desapercibidas para los líderes de los museos por muchas razones. Estos profesionales, como muchos otros relacionados con las tecnologías, tienen dificultades para hacer evidente el valor de sus contribuciones a los dirigentes debido a una falta general de comprensión acerca de la naturaleza de los proyectos relacionados con las tecnologías y las amplias responsabilidades que se asumen. Cuanto más quede entre bambalinas el trabajo de estos profesionales, y permanezca invisible, y además dejen de tener apoyo desde la dirección del museo, su labor se tornará todavía más intangible, y tendrán más probabilidades de que esto represente un peligro para los museos precisamente en crisis.

Como escribieron Nardi y Engeström (1999), “cuando las organizaciones se reestructuran y el trabajo se reorganiza, a menudo se elimina el trabajo invisible pero valioso. Nadie reconoce qué se está haciendo, o qué valor tiene, por lo que el tiempo y el personal necesarios no se asignan en nuevos planes" (p.1). Por ejemplo, existen malentendidos generales acerca de las responsabilidades de estos profesionales que pueden agravarse por la dificultad de transmitir indicadores tangibles de éxito en los proyectos (esto es particularmente cierto en situaciones en las que las lecciones aprendidas gracias a los fracasos pueden ser más valiosas que los resultados de un proyecto tecnológico en sí). De manera similar, la invisibilidad inherente del trabajo entre bastidores puede, lamentablemente, dar como resultado la percepción de que estos trabajadores no son esenciales para las operaciones del museo (después de todo, es fácil descartar lo que uno no ve, y casi todos los tecnólogos tienden a respetar la máxima de que, si algo es visible, significa que algo no ha funcionado).

Para muchos de estos profesionales, la invisibilidad inherente de su trabajo también va ligado a conceptos erróneos comunes sobre sus roles y responsabilidades cotidianas, especialmente 
en un mundo donde se supone que las infraestructuras que funcionan correctamente son invisibles. Por ejemplo, los malentendidos generales sobre el tiempo y el esfuerzo que requieren los proyectos tecnológicos pueden dar lugar a la creencia errónea de que la inversión en tecnología es excesivamente costosa y que dicha inversión proporciona poco retorno. Asimismo, la percepción errónea de que la divulgación en línea no contribuye directamente a las visitas presenciales (una métrica para definir el éxito en el mundo digital que continúa desafiando décadas de argumentos), esto puede dar como resultado una tendencia hacia la infravaloración del desarrollo tecnológico en los museos, en comparación con otras actividades.

Cuanto más invisible e infravalorado sea el trabajo cotidiano realizado entre bastidores por parte de estos profesionales, es menos probable que los dirigentes de museos dén prioridad a las iniciativas digitales. Incluso cuando los líderes solicitaron a los técnicos que apoyaran a sus instituciones durante la pandemia de COVID-19, seguía existiendo el temor subyacente entre ellos de que los avances aconteciesen en un lapso de tiempo corto. A muchos les preocupaba que mantener el foco sobre proyectos tecnológicos después de la pandemia pudiera convertirse un desafío, precisamente debido a los conceptos erróneos inherentes y los malentendidos cosechados alrededor del uso de las tecnologías en los museos. Si las lecciones aprendidas de la pandemia de COVID-19 no fueran a perderse, los técnicos de los museos podrían mejorar respecto a la defensa de su propio trabajo, para que los dirigentes de los museos tengan más probabilidades de priorizar las iniciativas digitales en el futuro.

\section{Abogar por las contribuciones de los profesionales de las tecnologías de los museos}

Para superar estos desafíos, los profesionales de las tecnologías deben defender con más fuerza el valor de sus contribuciones respecto a los dirigentes de los museos, tanto en tiempos de crisis como en tiempos estables. Esto se puede lograr al involucrar de forma constante a los líderes en el perpetuo trabajo de esta ocupación técnica y que se trabaje para demostrar su valor como comunidad profesional durante situaciones de crisis. Es imperativo que aprovechen todas las oportunidades disponibles para documentar su trabajo y proporcionar evidencias sobre sus beneficios respecto al museo, especialmente en un mundo donde el trabajo invisible que tiene lugar entre bambalinas puede darse por sentado con demasiada frecuencia.

Como escribió Marty (2016), “Mientras la gente crea que el fácil acceso a las colecciones del museo simplemente apareció por arte de magia, no gracias a su trabajo invisible, siempre existe el riesgo de que alguien pregunte: “¿Por qué eres importante? ¿Cuáles fueron tus aportaciones? y ¿quién eres tú de todos modos?” (p.20). Estos profesionales pueden luchar contra esta tendencia, por ejemplo, haciendo más visibles sus actividades cotidianas desarrolladas encubiertamente, y también compartiendo de manera proactiva ejemplos de proyectos tecnológicos exitosos con un público más amplio (el proyecto que es no compartido se convierte fácilmente en un proyecto que nadie conoce y equivale a un proyecto 
que no aconteció). De manera similar, proporcionar métricas que documenten la capacidad de las tecnologías interactivas para mejorar las actividades y la participación de la audiencia puede ayudar a demostrar el valor de las iniciativas de divulgación en línea con respecto a las visitas físicas al museo.

También sería útil para ellos trabajar más de cerca con la dirección del museo mientras exploran nuevas estrategias digitales y promueven nuevos enfoques que ayuden a resolver problemas técnicos. Por ejemplo, pueden trabajar para involucrar a los líderes directamente en los proyectos tecnológicos en curso, alentándoles a tomar la iniciativa de documentar la necesidad y el valor de las estrategias digitales en tiempos de crisis. Del mismo modo, pueden unir fuerzas con otros trabajadores de otros museos, solicitando la ayuda de organizaciones profesionales que apoyan el uso de la tecnología en museos para documentar y difundir datos acerca del valor del trabajo de sus contribuciones, o a través de actividades como la realización de encuestas a los visitantes que determinen sus actitudes hacia el uso de las tecnologías interactivas en las salas y en línea.

Cuanto más se evidencia que estos profesionales ayudan a mejorar el liderazgo de los museos, más profesionales y tecnólogos podrán argumentar sus contribuciones y aumentar la comprensión general o la visibilidad de sus logros. Durante la pandemia de COVID-19, demostraron una y otra vez su capacidad para mantener los museos abiertos a la vista de sus visitantes virtuales, facilitando el acceso a las colecciones y siendo útiles y funcionales para los empleados que trabajaron remotamente, logrando que los museos fuesen relevantes para sus comunidades distribuidas, siendo de vital importancia que estos logros no pasen desapercibidos. Al proporcionar evidencia de la eficacia de sus esfuerzos de divulgación y el valor de su trabajo para los diversos interesados en los museos, no solo los profesionales de las tecnologías tienen la oportunidad de hacer más visibles sus propias contribuciones, sino que los dirigentes también tienen la oportunidad de promover e invertir en las contribuciones de su personal.

\section{Invertir en las contribuciones de los profesionales de las tecnologías de los museos}

Existe una clara necesidad de que los dirigentes de los museos inviertan en la alfabetización digital y en las estrategias digitales necesarias para afrontar las crisis del siglo XXI. Es hora de que los líderes de los museos reconozcan que estos profesionales son tan esenciales como los comisarios para el correcto funcionamiento del museo moderno. A medida que los museos avanzan hacia un mundo posterior a la COVID, no deberían esforzarse por volver a la "vieja normalidad", sino que deberían aprovechar esta oportunidad para repensar su rol, y centrarse en las posibilidades de utilizar la tecnología para remodelar la experiencia museística, y buscar una "nueva normalidad" que sea mejor para todos los profesionales de los museos.

En un mundo donde lo digital lo impregna todo, no hay futuro para los museos que no tienen empleados con habilidades digitales, la mentalidad digital y las estrategias digitales que los 
museos necesitan para sobrevivir. Los profesionales de las tecnologías de los museos están en una posición única para proporcionar el liderazgo digital que todos los museos necesitan, pero que pocos tienen, precisamente durante situaciones de crisis. Como informó la Fundación Knight (2020), “A medida que más museos comiencen a hacer de la participación de la audiencia digital una prioridad, aumentará la necesidad de personal enfocado en lo digital. [Pero ...] la mitad de las instituciones encuestadas informan que no cuentan con ninguna persona, o a lo sumo, con una sola persona como personal digital".

Es de vital importancia que los profesionales de los museos desarrollen habilidades en áreas como la gestión de contenido, la alfabetización en medios digitales, la experiencia del usuario, la ética de los datos, la seguridad o la política de la información. Afortunadamente, hay muchas organizaciones profesionales y grupos académicos que trabajan para desarrollar estas habilidades y abordar esta brecha de habilidades críticas (Parry et al., 2018). Desafortunadamente, no importa cuán entusiastas puedan ser los museos en esta nueva normalidad, uno de los mayores problemas a los que se enfrenta hoy en día la informática de los museos es la dificultad que tienen para aferrarse a técnicos capacitados. Como escribe Chan (2020), "con el éxodo masivo de talentos técnicos y calificados del sector, [...] hay muy pocas personas con la profundidad y la experiencia en el sector que puedan ayudar a los museos a tomar decisiones tecnológicas estratégicas sensatas”.

Si los museos quieren revertir esa tendencia, entonces, en lugar de despedir a las personas que están mejor situadas para ayudarles a tomar decisiones estratégicas, deberían adoptar las habilidades de alfabetización digital necesarias en sus empleados y aumentar su inversión en tecnología, en línea y presencialmente. Para que los museos "dejen de hacer las cosas mal”, los líderes y los profesionales de las tecnologías deben trabajar juntos para enfrentar con éxito los desafíos futuros y sobrevivir a futuras crisis. A través de una comprensión compartida de sus contribuciones en tiempos de crisis, estos profesionales junto a los líderes de los museos podrán posicionar mejor a sus instituciones con las habilidades digitales, la mentalidad y las estrategias que todos los museos necesitarán para tener éxito en el futuro.

\section{Referencias}

American Alliance of Museums (AAM). (2020). National survey of COVID-19 impact on United States museums. https://www.aam-us.org/2020/07/22/a-snapshot-of-usmuseums-response-to-the-covid-19-pandemic/

Anderson, S. (2018). Visitor and audience research in museums. In K. Drotner, V. Dziekan, R. Parry, \& K.C. Schroder (Eds.), The Routledge Handbook of Museums, Media and Communication. London: Taylor and Francis, Inc.

Chan, S. (2020). Looking backwards to go forward - words from talks in late 2020. Retrieved from https://sebchan.medium.com/looking-backwards-to-go-forward-wordsfrom-talks-in-late-2020-b20f90ce6375 
Evjen, M. (2020, July 22). Tweet. https://twitter.com/cantus94/status/1286136406948052992

Hart, T., Brooks, C., Spellerberg, M., \& Poole, G. (2020). Effects Of Shelter-In-Place On Museum Website Visitation: Findings From A Cross-institutional Study. Museum Computer Network 2020 Conference. November 10, 2020. Retrieved from https://mcn.edu/conferences/mon-2020-virtual/

International Council of Museums (ICOM). (2013). Support culture and museums to face the global crisis and build the future. https://icom.museum/wpcontent/uploads/2018/07/Lisbon_Declaration_ENG.pdf

International Council of Museums (ICOM). (2020). Museums, museum professionals and COVID-19. https://icom.museum/wp-content/uploads/2020/05/Report-Museums-andCOVID-19.pdf

Knight Foundation. (2020). Digital readiness and innovation in museums: A baseline national survey. https://knightfoundation.org/reports/digital-readiness-and-innovationin-museums/

Marty, P.F. (2016). The cobbler's children: Invisible work and information professionals in museums. In J.W. Arns (Ed.), Annual Review of Cultural Heritage Informatics (pp.1922). Lanham: Rowman and Littlefield.

McGrath, J. (2020). Museums and social media during COVID-19. The Public Historian, 42(4), 164-172.

Nardi, B., \& Engeström, Y. (1999). A web on the wind: The structure of invisible work. Computer Supported Cooperative Work, 8(1), 1-2.

Parry, R., Eikhof, D.R., Barnes, S.-A., \& Kispeter, E. (2018). Development, supply, deployment, demand: Balancing the museum digital skills ecosystem. First findings of the 'One by One' national digital literacy project. Proceedings of the Museums and the Web Conference 2018. https://mw18.mwconf.org/paper/development-supplydeployment-demand-balancing-the-museum-digital-skills-ecosystem-first-findings-ofthe-one-by-one-national-digital-literacy-project/

Small, Z. (2020, September 15). 'The pandemic was just the final straw': The hard-hit arts sector is facing a brain drain as ambitious workers seek greener pastures. Art Net News. https://news.artnet.com/art-world/art-industry-brain-drain-1907518

United Nations Educational, Scientific and Cultural Organization (UNESCO). (2020). Museums around the world in the face of COVID-19. https://unesdoc.unesco.org/ark:/48223/pf0000373530Van Goch, M. (2018). Creativity in liberal education before and after study commencement. 4th International Conference on Higher Education Advances (HEAd'18), 1475-1483. doi: 10.4995/HEAd18.2018.8228. 